\title{
Erythrocyte Omega-3, Omega-6 and Trans Fatty Acids in Relation to Risk of Preeclampsia among Women Delivering at Harare Maternity Hospital, Zimbabwe
}

\author{
K. MAHOMED ${ }^{1}$, M. A. WILLIAMS ${ }^{2-4}$, I. B. $\mathrm{KING}^{4}$, S. MUDZAMIRI ${ }^{1}$, \\ G. B. WOELK ${ }^{5}$
}

${ }^{I}$ Departments of Obstetrics and Gynecology and ${ }^{5}$ Community Medicine, University of Zimbabwe, School of Medicine, Harare, Zimbabwe, ${ }^{2}$ Department of Epidemiology, University of Washington, School of Public Health and Community Medicine, ${ }^{3}$ Center for Perinatal Studies, Swedish Medical Center, ${ }^{4}$ Public Health Sciences Division, Fred Hutchinson Cancer Research Center, Seattle, Washington, USA

Received September 8, 2005

Accepted February 20, 2006

On-line available February 23, 2006

\begin{abstract}
Summary
We sought to examine the association between maternal erythrocyte omega-3, omega- 6 and trans fatty acids and risk of preeclampsia. We conducted a case-control study of 170 women with proteinuric, pregnancy-induced hypertension and 185 normotensive pregnant women who delivered at Harare Maternity Hospital, Harare, Zimbabwe. We measured erythrocyte omega-3, omega- 6 and trans fatty acid as the percentage of total fatty acids using gas chromatography. After multivariate adjustment for confounding factors, women in the highest quartile group for total omega-3 fatty acids compared with women in the lowest quartile experienced a $14 \%$ reduction in risk of preeclampsia (odds ratio 0.86 , $95 \%$ confidence interval 0.45 to 1.63 ). For total omega- 6 fatty acids the odds ratio was 0.46 ( $95 \%$ confidence interval 0.23 to 0.92 ), although there was suggestion of a slight increase in risk of preeclampsia associated with high levels of arachidonic acid. Among women in the highest quartile for arachidonic acid the odds ratio was 1.29 (95\% confidence interval 0.66 to 2.54). A strong statistically significant positive association of diunsaturated fatty acids with a trans double bond with risk of preeclampsia was observed. Women in the upper quartile of 9-cis 12-trans octadecanoic acid $\left(\mathrm{C}_{18: 2 n 6 c t}\right)$ compared with those in the lowest quartile experienced a 3-fold higher risk of preeclampsia (odds ratio $=3.02$, $95 \%$ confidence interval 1.41 to 6.45 ). Among women in the highest quartile for 9-trans 12-cis octadecanoic acid $\left(\mathrm{C}_{18: 2 n \text { ntc }}\right)$ the odds ratio was 3.32 (95\% confidence interval 1.55 to 7.13$)$. Monounsaturated trans fatty acids were also positively associated with the risk of preeclampsia, although of much reduced magnitude. We observed a strong positive association of trans fatty acids, particularly diunsaturated trans fatty acids, with the risk of preeclampsia. We found little support for the hypothesized inverse association between omega-3 fatty acids and preeclampsia risk in this population. Polyunsaturated fatty acids, particularly omega-3 fatty acids, were comparatively lower in Zimbabwean than among US pregnant women. Given the limited inter-person variation in omega-3 fatty acids among Zimbabwean women, our sample size may be too small to adequately assess the relation in this population.
\end{abstract}




\section{Key words}

Diet $\bullet$ Omega-3 fatty acids $\bullet$ Omega- 6 fatty acids $\bullet$ Preeclampsia $\bullet$ trans fatty acids

\section{Introduction}

Habitual consumption of modest amounts of fish and other seafood has been associated with a reduction in the risk of heart disease (Harris 2005). Results from controlled metabolic studies have provided strong evidence for the role of fish oil (i.e., long chain omega-3 polyunsaturated fatty acids) played in reducing circulating triglycerides, decreasing platelet and leukocyte reactivity, attenuating imbalances between the potent eicosanoids such as prostacyclin and thromboxane in favor of vasodilatation, and reducing systolic and diastolic blood pressures (Harris 2005, Kinsella et al. 1990). The beneficial effects of fish oil appear to be associated with the long chain polyunsaturated omega-3 fatty acids - eicosapentaenoic, docosapentaenoic and docosahexaenoic acids. Together these fatty acids represent the main omega-3 polyunsaturated fatty acids in the diet. Although derived primarily from fatty fish and some shellfish, omega-3 polyunsaturated fatty acids can also be derived from the elongation and desaturation of $\alpha$-linolenic acid, an omega- 3 fatty acid derived from certain vegetables, seeds and nuts (Kestin et al. 1990). Omega-6 fatty acids are derived from vegetables (sunflower, corn and cotton seed), eggs and other animal sources (Kestin et al. 1990). Arachidonic acid, an omega6 fatty acid, has been shown to have opposite effects of omega-3 fatty acids on circulating lipids, platelet and leukocyte reactivity, as well as eicosanoid biosynthesis (Kinsella et al. 1990, Kestin et al. 1990).

Trans fatty acids, derived primarily from the partial hydrogenation of vegetable oils to produce margarines, salad oils, shortening and a variety of prepared foods, have been associated with increased coronary heart disease risk in some, though not all studies (Hu et al. 1997, Aro et al. 1995, Roberts et al. 1995). Trans fatty acids have been found to be associated with an adverse plasma lipid profile (Katan and Zock 1995), inhibition of the desaturation and elongation of essential omega- 3 and omega- 6 fatty acids, and increased platelet aggregation. Investigators have documented a positive association between trans fatty acids and plasma lipoprotein(a) [Lp(a)] concentrations (Katan and Zock 1995).

Preeclampsia, one of the most common medical complications of pregnancy (Roberts et al. 2003), is a leading cause of maternal mortality worldwide, as well as an important cause of premature delivery, fetal growth retardation, and perinatal mortality. Pregnancies complicated by preeclampsia are preceded by hyperlipidemia, particularly hypertriglyceridemia. Other pathologic changes associated with preeclampsia include maladaptation of spiral arteries of the placental bed, excessive lipid peroxidation, endothelial cell dysfunction, thromboxane/prostacyclin imbalance, elevations in proinflammatory cytokines, altered membrane fluidity, and elevations in plasma homocyst(e)ine concentrations (Williams and Mittendorf 2000, Roberts et al. 2003, Qiu et al. 2006). Controlled metabolic studies provided evidence suggesting that manipulation of the dietary intake of omega- 3 fatty acids, omega- 6 fatty acids, and trans fatty acids may prevent or ameliorate several of these pathophysiological processes (Katan and Zock 1995, Harris 2005).

Investigators have previously shown that derangements in omega- 3 and omega- 6 fatty acids in plasma phospholipids or erythrocytes are associated with preeclampsia and other adverse pregnancy outcomes (Ogburn et al. 1984, Olsen et al. 1991, Williams et al. 1995, Clausen et al. 2001). Very little is known, however, about the metabolic consequence of dietary intake of trans fatty acids in pregnancy. At least one group of investigators, however, have reported that elaidic acid, one of the major dietary trans fatty acids, was inversely associated with infant birth weight (Koletzko 1992). In a small cross-sectional pilot study conducted among predominately white women in Seattle (Washington, USA) we observed a strong positive association between maternal erythrocyte elaidic acid and risk of preeclampsia (Williams et al. 1998).

For the present study, we examined the association between maternal erythrocyte composition of omega-3 and omega-6, as well as trans fatty acids in relation to risk of preeclampsia among women in Harare, Zimbabwe. Given that erythrocyte turnover is approximately 120 days, and that erythrocyte fatty acid profiles have been shown to reflect relative patterns of dietary intake of fats from marine and vegetable sources over a 2- to 3-month period (Farquhar and Ahrens 1983), we decided to assess fatty acid profiles in maternal erythrocytes. 


\section{Methods}

\section{Study Setting and Population}

This case-control study was conducted at Harare Maternity Hospital, a University of Zimbabwe Medical School affiliated hospital, from June 1995 through April 1996. Harare Maternity Hospital is a referral hospital that is part of what is known as the Greater Harare Maternity Unit. The Unit, comprised of Harare Maternity Hospital and nine clinics located in Harare's high density suburbs, is responsible for delivering 36000 infants annually.

During the study period, we sought to enroll 200 women with preeclampsia or eclampsia and 200 normotensive control women. Potential cases were identified by daily surveillance of labor and delivery log books and medical records. Study subjects were recruited during their postpartum stay in the hospital. Preeclampsia was defined as a sustained $15 \mathrm{mmHg}$ diastolic rise or a $30 \mathrm{mmHg}$ rise in systolic blood pressure above prepregnant or first trimester levels. If first trimester blood pressures were unknown, preeclampsia was defined as persistent (6 or more hours) blood pressure of at least 140/90 $\mathrm{mm} \mathrm{Hg}$. Proteinuria was defined as urine protein concentration of $0.1 \mathrm{~g} / 1$ or more in at least two random specimens collected at least $4 \mathrm{~h}$ apart. Eclampsia was defined as above with the additional stipulation of maternal seizures (ACOG 1996). Nulliparity was not a criterion for diagnosis for this investigation. All 200 eligible cases who were approached agreed to participate in the study. Cases were comprised of 37 patients with eclampsia and 163 patients with preeclampsia. There were 17028 deliveries at the Hospital during the study period, approximately $4.3 \%$ of deliveries were complicated by preeclampsia $(n=621)$ or eclampsia $(n=107)$.

Controls were women with pregnancies uncomplicated by pregnancy-induced hypertension. Each day during the enrollment period, controls were numbered in the order in which they were admitted and delivered within two hours of a case. If a selected control was too ill to participate, the next eligible control on the list was approached and recruited. Of the 201 controls approached, 200 (99\%) agreed to participate in the study. During the study period, only three women selected for potential participation in the study was characterized as being too ill (i.e., not able to participate in the study interview because of physical fatigue or emotional distress). Recruitment of cases and controls took place during the regular day shift of a 5-day (Monday through
Friday) work week.

Women with pre-gestational chronic hypertension were not eligible for inclusion in the present study. Blood samples were available for 190 cases and 191 control subjects. After excluding 20 cases and 6 control subjects with chronic hypertension diagnosed prior to pregnancy or during the first 20 weeks of the index pregnancy, 170 preeclampsia or eclampsia cases (32 eclamptics and 138 preeclamptics) and 185 normotensive control subjects remained for the study. The case group will be referred to as preeclamptics. This investigation was approved by the Medical Research Council of Zimbabwe and the Human Subjects Committee of the University of Washington Medical Center. All subjects provided informed consent before participating in the study.

\section{Data collection}

A structured interview questionnaire was administered during participants' postpartum hospital stay and information concerning antepartum, labor and delivery characteristics, and conditions of the newborn were obtained from medical records. Maternal anthropometric measures (height, weight and mid-arm circumference) were taken during participants' postpartum hospital stay. Because pre-pregnancy weight is difficult to obtain in developing countries (Krasoveck et al. 1991), and because Zimbabwean women are unlikely to be able to report their pre-pregnancy weight, we elected $a$ priori to use maternal mid-arm circumference, measured at delivery, as our primary measure of maternal pre-pregnancy adiposity. Maternalmid-arm circumference was taken from participants' nondominant arm. The circumference of the mid-arm (point mid-way between the elbow and shoulder) was measured using a cloth tape measure. All measurements were taken by one author (SM).

\section{Specimen collection and processing}

Blood samples collected in EDTA $10 \mathrm{ml}$ Vacutainer tubes $12-72 \mathrm{~h}$ postpartum, were immediately transported, in a cooler with ice, to the Reproductive Biology Laboratory, Parirenyatwa Hospital, University of Zimbabwe Medical School. Upon arrival at the laboratory, plasma was separated and erythrocytes were washed three times. Washed erythrocytes and plasma were divided into 1-2 $\mathrm{ml}$ aliquots, placed in cryovials, and stored at $-70{ }^{\circ} \mathrm{C}$. Specimens were shipped in liquid nitrogen to the USA for biochemical analyses. 


\section{Biochemical analyses}

Fatty acid methyl esters (FAME) were prepared by direct trans-esterification using the method of Lepage and Roy (1986). Gas chromatography was performed on samples dissolved in hexane. The FAME of total erythrocyte fatty acids were separated on a gas chromatograph (model 5890B, series II, Hewlett-Packard, Avondale, PA) equipped with a flame ionization detector (FID), automatic sampler (Hewlett-Packard 7673) and Chemstation software (Hewlett-Packard). Quantitative precision, accuracy and identification of fatty acids were evaluated using the model mixtures of known FAME and an established erythrocyte specimen control pool. Quantitative results were standardized with the National Heart Institute's Fatty Acid Standards A-D, F, and GLC87 (Nu-Check-Prep, Elysian, MN). Quantification was made by integrating the peak area of each FAME (using HP Chemstation software), and results were expressed as relative weight percentage. Erythrocyte membrane composition of eicosapentaenoic acid (an omega-3 fatty acid), for example, was expressed as the mean weight percentage $( \pm \mathrm{SD})$. Inter-assay coefficients of variation $(\mathrm{CV})$, determined by analysis of aliquots from a pooled sample, were 7, 3 and $2 \%$ for eicosapentaenoic acid, docosahexaenoic acid, and arachidonic acid, respectively. Inter-assay CV's ranged from 2-7 \% for monounsaturated trans fatty acids, $13-25 \%$ for diunsaturated fatty acids with a trans double bond. All laboratory analyses were blinded as to case or control status. A total of 41 fatty acids were identified. Below we report on only those fatty acids (i.e. omega-3, omega-6 and trans fatty acids) for which we had hypothesized would be associated with the occurrence of preeclampsia.

\section{Statistical analysis}

The frequency distribution of maternal sociodemographic characteristics, medical and reproductive histories according to case control status was examined. Because comparison of group means (or other measures of central tendency) may obscure important group differences in the tails of distributions, we elected a priori to assess the relation of each fatty acid and risk of preeclampsia after creating ordinal categorical variables. We also compared group mean differences for each fatty acid studied. To estimate the relative association between preeclampsia and levels of specific fatty acids, we categorized each subject according to quartiles determined by the distribution of fatty acid levels in controls (Hsieh et al. 1991). Using the lowest fatty acid category as the referent group, odds ratios and their attendant $95 \%$ confidence intervals were estimated. Logistic regression procedures were used to calculate maximum likelihood estimates for the coefficients and their standard errors were used to calculate odds ratios and $95 \%$ confidence intervals (CI), adjusted for confounders (Rothman and Greenland 1998). In multiple logistic regression models, significance for monotonic trends was assessed by treating the four quartiles as a continuous variable after assigning a score as its value (Rothman and Greenland 1998). To assess confounding, we entered variables into a logistic regression model one at a time, then compared the adjusted and unadjusted odds ratios. Final logistic regression models included covariates that altered unadjusted ORs by at least $10 \%$. Results of analyses restricted to preeclamptics (i.e. excluding the 32 eclamptics) were not materially different from those results, which included all 170 case subjects. Hence, we report results for preeclampsia and eclampsia cases combined. All reported $p$ values are two tailed.

\section{Results}

Comparison of the group mean differences of maternal mid-arm circumference was consistent with cases being heavier than controls. Comparisons of group mean differences for omega- 3 fatty acids, omega- 6 fatty acids and trans fatty acids are presented in Table 1.

Unadjusted and adjusted odds ratios of preeclampsia for each quartile of omega-3 fatty acids are presented in Table 2. Overall, we observed no clear evidence of a linear trend in risk of preeclampsia with increasing levels of omega- 3 fatty acids. The adjusted odds ratios and $95 \% \mathrm{CI}$ for increasing quartiles of linolenic acid $\left(\mathrm{C}_{18: 3 n 3}\right)$, were $(\mathrm{OR}=1.42,95 \% \mathrm{CI} 0.73$ 2.76; $\mathrm{OR}=0.70,95 \% \mathrm{CI} 0.35-1.41$; and $\mathrm{OR}=0.86,95 \%$ CI $0.43-1.69$, respectively) compared with the lowest quartile (trend $p$-value $=0.29$ ). There appeared to be a reverse J-shaped relation between the sum of omega-3 fatty acids and risk of preeclampsia. Women in the second quartile of total omega-3 fatty acids, as compared to those in the lowest quartile group, had a $58 \%$ reduction in risk of preeclampsia (adjusted $\mathrm{OR}=0.42$ $95 \%$ CI $0.20-0.87$ ). Preeclampsia risk appeared to be reduced for women in the third and fourth quartiles, although the reduction in risk was attenuated (ORs $=0.77$ and 0.86 , respectively) and did not reach statistical significance (Fig. 1).

Results from analyses of preeclampsia risk in 
Table 1. Percentages of omega- 3 and omega- 6 polyunsaturated fatty acids and trans fatty acids in erythrocytes obtained from preeclamptic-eclamptic cases and normotensive controls, Harare, Zimbabwe 1995-1996.

\begin{tabular}{|c|c|c|c|}
\hline Fatty Acid & $\begin{array}{l}\text { Preeclampsia Cases } \\
(\mathbf{n}=170) \\
\text { Mean } \pm \text { SD }\end{array}$ & $\begin{array}{l}\text { Control Subjects } \\
(n=185) \\
\text { Mean } \pm \text { SD }\end{array}$ & p value \\
\hline \multicolumn{4}{|l|}{ Omega-3 fatty acids } \\
\hline Linolenic acid $\left(C_{18: 3 n 3}\right)$ & $0.17 \pm 0.06$ & $0.18 \pm 0.06$ & 0.2 \\
\hline Eicosapentaenoic acid $\left(C_{20: 5 n 3}\right)$ & $0.20 \pm 0.07$ & $0.20 \pm 0.07$ & 0.47 \\
\hline Docosapentaenoic acid $\left(C_{22: 5 n 3}\right)$ & $1.63 \pm 0.20$ & $1.64 \pm 0.21$ & 0.82 \\
\hline Docosahexaenoic acid $\left(C_{22: 6 n 3}\right)$ & $5.19 \pm 0.81$ & $5.03 \pm 0.77$ & 0.06 \\
\hline Sum Omega-3 fatty acids & $7.18 \pm 0.92$ & $7.05 \pm 0.88$ & 0.15 \\
\hline \multicolumn{4}{|l|}{ Omega-6 fatty acids } \\
\hline Linoleic acid $\left(C_{18: 2 n 6}\right)$ & $10.38 \pm 1.44$ & $10.74 \pm 1.26$ & 0.01 \\
\hline$\gamma$-Linoleic acid $\left(C_{18: 3 n \sigma}\right)$ & $0.25 \pm 0.14$ & $0.25 \pm 0.14$ & 0.87 \\
\hline Eicosadienoic acid $\left(C_{20: 2 n 6}\right)$ & $0.47 \pm 0.08$ & $0.49 \pm 0.08$ & 0.06 \\
\hline Eicosatrienoic acid $\left(C_{20: 3 n \sigma}\right)$ & $1.42 \pm 0.22$ & $1.43 \pm 0.19$ & 0.57 \\
\hline Arachidonic acid $\left(C_{20: 4 n \sigma}\right)$ & $12.74 \pm 0.94$ & $12.76 \pm 0.91$ & 0.85 \\
\hline Docosatetraenoic acid $\left(C_{22: 4 n}\right)$ & $3.86 \pm .052$ & $4.00 \pm 0.54$ & 0.02 \\
\hline Sum Omega-6 fatty acids & $29.19 \pm 1.38$ & $29.75 \pm 1.28$ & $<0.001$ \\
\hline Ratio of Omega-3/Omega 6 & $0.25 \pm 0.04$ & $0.24 \pm 0.04$ & 0.02 \\
\hline Ratio of Omega-3/Arachidonic acid & $0.57 \pm 0.08$ & $0.56 \pm 0.08$ & 0.02 \\
\hline \multicolumn{4}{|l|}{$C_{18}$ monounsaturated trans fatty acids } \\
\hline 8, 7,6-trans octadeceonic acid $\left(C_{18: \ln 10-12 t}\right)$ & $0.07 \pm 0.06$ & $0.07 \pm 0.06$ & 0.43 \\
\hline 9-trans octadecanoic acid $\left(C_{18: \ln 9 t}\right)$ & $0.16 \pm 0.06$ & $0.15 \pm 0.08$ & 0.15 \\
\hline 10-trans octadecanoic acid $\left(C_{18: \ln 8 t}\right)$ & $0.14 \pm 0.06$ & $0.12 \pm 0.06$ & 0.002 \\
\hline 11-trans octadecanoic acid $\left(C_{18: \ln 7 t}\right)$ & $0.22 \pm 0.07$ & $0.21 \pm 0.09$ & 0.41 \\
\hline 12-trans octadecanoic acid $\left(C_{18: \ln 6 t}\right)$ & $0.27 \pm 0.06$ & $0.26 \pm 0.05$ & 0.06 \\
\hline Sum Monounsaturated trans fatty acids & $0.86 \pm 0.24$ & $0.81 \pm 0.24$ & 0.05 \\
\hline \multicolumn{4}{|c|}{ Diunsaturated fatty acids with a trans double bond } \\
\hline 9-cis 12-trans octadecanoic acid $\left(C_{18: 2 n \sigma c t}\right)$ & $0.05 \pm 0.02$ & $0.05 \pm 0.03$ & 0.24 \\
\hline 9-trans 12-cis octadecanoic acid $\left(C_{18: 2 n o t c}\right)$ & $0.04 \pm 0.01$ & $0.03 \pm 0.01$ & $<0.001$ \\
\hline Sum Diunsaturated trans fatty acids & $0.09 \pm 0.03$ & $0.08 \pm 0.03$ & 0.002 \\
\hline
\end{tabular}

relation to omega-6 fatty acid levels are presented in Table 3. There was a modest, though statistically insignificant, reduction in risk of preeclampsia with increasing levels of linoleic acid $\left(\mathrm{C}_{18: 2 n 6}\right)$ (adjusted p-for trend $=0.23$ ). There was a $30 \%$ reduction in the risk of preeclampsia for women in the upper quartile, as compared to those in the lowest quartile $(\mathrm{OR}=0.70$, $95 \%$ CI 0.36-1.37). A similar pattern was seen for most of the other omega- 6 fatty acids studied, although a statistically significant inverse linear trend in the risk was seen for docosatetraenoic acid $\left(\mathrm{C}_{22: 4 n 6}\right)$ (adjusted p-for trend $=0.04)$. Odds ratios corresponding to successively higher quartiles of arachidonic acid $\left(\mathrm{C}_{20: 4 n 6}\right)$ were 1.19
(95\% CI 0.60-2.23), 0.79 (95\% CI 0.29-2.33) and 1.29 (95\% CI 0.66-2.54), with the lowest quartile as the referent group. There was a linear trend in decreasing risk of preeclampsia with increasing levels of the sum of omega- 6 fatty acids. The odds ratios and $95 \% \mathrm{CI}$ for increasing quartiles of total omega- 6 fatty acids were 0.85 (95\% CI 0.45-1.61), $0.66(0.35-1.33)$ and 0.46 (95\% CI 0.23-0.92), compared with the lowest quartile as the referent group (adjusted $\mathrm{p}$ for trend $=0.02$ ).

Because omega-3 fatty acids interact with fatty acids in the omega- 6 family in eicosanoid biosynthesis, and because omega-3 fatty acids are rapidly and reversibly incorporated into membranes of erythrocytes, 
Table 2. Odds Ratios (OR) and $95 \%$ Confidence Intervals (CI) of preeclampsia according to quartile of percentages of omega-3 polyunsaturated fatty acids in maternal erythrocytes, Harare, Zimbabwe, 1995-1996.

\begin{tabular}{|c|c|c|c|c|c|}
\hline \multirow[b]{2}{*}{ Fatty Acid } & \multicolumn{4}{|c|}{ Quartile of Measurement } & \multirow[b]{2}{*}{$\begin{array}{l}\text { trend } \\
\text { p-value }\end{array}$} \\
\hline & 1 (Low) & 2 & 3 & 4 (High) & \\
\hline \multicolumn{6}{|c|}{ Linolenic acid $\left(C_{18: 3 n 3}\right)$} \\
\hline Mean & 0.1 & 0.15 & 0.20 & 0.26 & \\
\hline No. cases & 51 & 48 & 35 & 36 & \\
\hline No. controls & 47 & 46 & 46 & 46 & \\
\hline OR & 1 & 0.96 & 0.70 & 0.72 & 0.17 \\
\hline $\operatorname{Adj} O R^{*}(95 \% C I)$ & 1 & $1.42(0.73-2.76)$ & $0.70(0.35-1.41)$ & $0.86(0.43-1.69)$ & 0.29 \\
\hline \multicolumn{6}{|c|}{ Eicosapentaenoic acid $\left(C_{20: 5 n 3}\right)$} \\
\hline Mean & 0.13 & 0.17 & 0.22 & 0.29 & \\
\hline No. cases & 58 & 24 & 53 & 35 & \\
\hline No. controls & 47 & 47 & 46 & 45 & \\
\hline OR & 1 & 0.41 & 0.93 & 0.63 & 0.41 \\
\hline $\operatorname{Adj} O R^{*}(95 \% C I)$ & 1 & $0.39(0.27-0.54)$ & $0.73(0.39-1.37)$ & $0.58(0.30-1.13)$ & 0.23 \\
\hline \multicolumn{6}{|c|}{ Docosapentaenoic acid $\left(C_{22: 5 n 3}\right)$} \\
\hline Mean & 1.39 & 1.57 & 1.69 & 1.91 & \\
\hline No. cases & 44 & 44 & 46 & 36 & \\
\hline No. controls & 48 & 45 & 46 & 46 & \\
\hline OR & 1 & 1.07 & 1.09 & 0.85 & 0.66 \\
\hline $\operatorname{Adj}_{O R^{*}}^{*}(95 \% C I)$ & 1 & $1.03(0.54-2.01)$ & $0.74(0.38-1.45)$ & $0.63(0.31-1.25)$ & 0.12 \\
\hline \multicolumn{6}{|c|}{ Docosahexaenoic acid $\left(C_{22: 6 n 3}\right)$} \\
\hline Mean & 4.16 & 4.70 & 5.14 & 6.08 & \\
\hline No. cases & 34 & 29 & 46 & 61 & \\
\hline No. controls & 47 & 46 & 46 & 46 & \\
\hline OR & 1 & 0.87 & 1.38 & 1.83 & 0.01 \\
\hline $\operatorname{Adj} O R^{*}(95 \% C I)$ & 1 & $0.69(0.33-1.41)$ & $1.12(0.57-2.21)$ & $1.20(0.62-2.34)$ & 0.34 \\
\hline \multicolumn{6}{|c|}{ Sum Omega-3 fatty acids } \\
\hline Mean & 6.06 & 6.71 & 7.20 & 8.20 & \\
\hline No. cases & 44 & 24 & 41 & 61 & \\
\hline No. controls & 47 & 46 & 46 & 46 & \\
\hline OR & 1 & 0.56 & 0.95 & 1.42 & 0.09 \\
\hline $\operatorname{Adj} O R^{*}(95 \% C I)$ & 1 & $0.42(0.20-0.87)$ & $0.77(0.39-1.50)$ & $0.86(0.45-1.63)$ & 0.99 \\
\hline
\end{tabular}

*Adjusted for maternal age, parity, mid-arm circumference, and gestational age at delivery.

largely at the expense of omega- 6 fatty acids, we also evaluated the ratio of the total level of omega- 3 fatty acids to the total level of omega- 6 fatty acids. We calculated the ratio of total omega-3 fatty acids:arachidonic acid ratio so as to compare our results with those of Olsen et al. (1991). After adjusting for confounders, women in the upper two quartiles of the omega-3:omega-6 ratio, as compared with those in the lowest quartile, appeared to be at an increased risk of preeclampsia, although the odds ratios did not reach statistical significance and the adjusted $95 \%$ confidence intervals are wide.

Risk of preeclampsia was moderately increased with most, although not all, of the $\mathrm{C}_{18}$ monounsaturated trans fatty acids measured in this population (Table 4). Among this group of trans fatty acids, maternal 


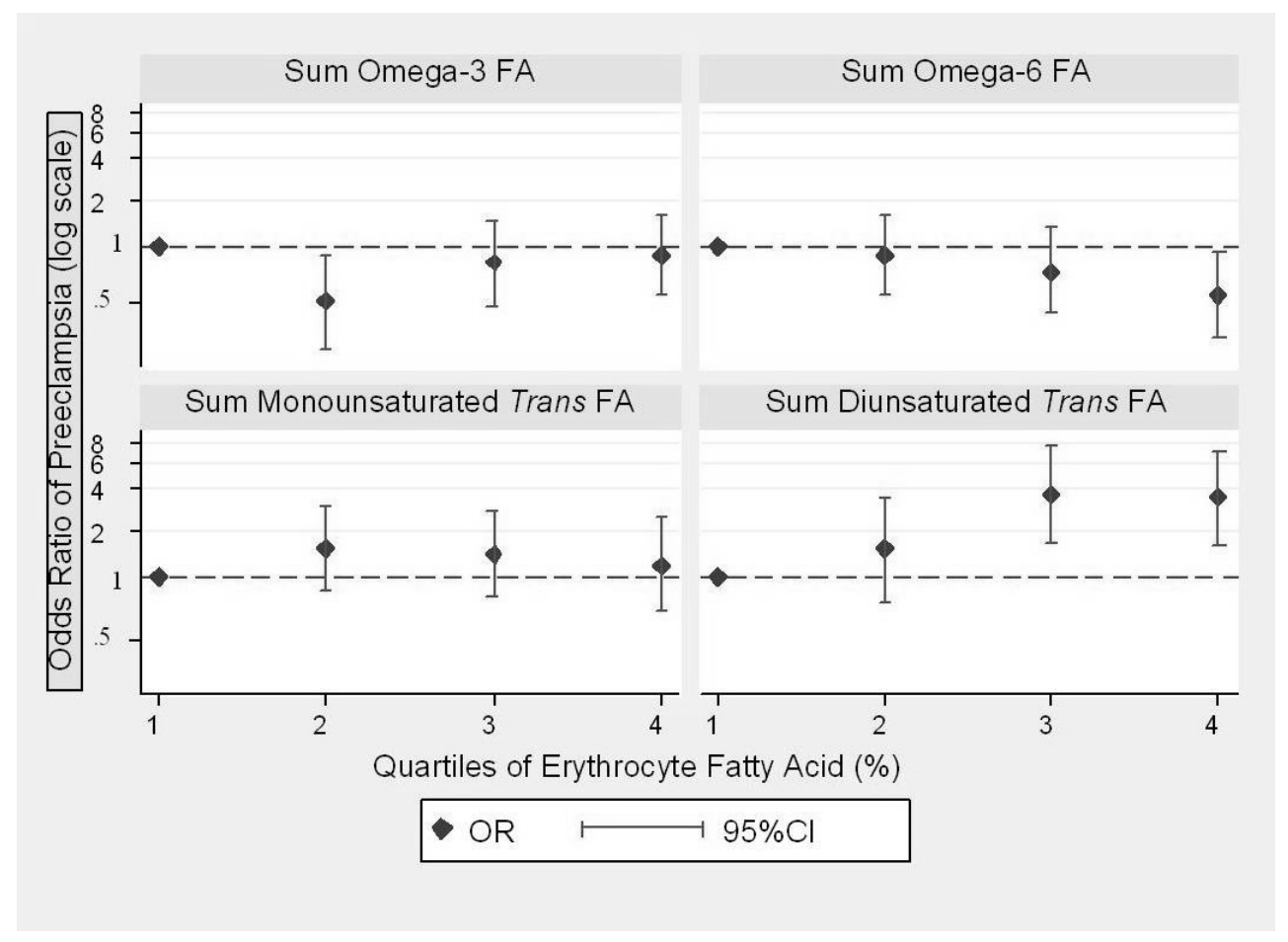

Fig. 1. Odds ratios (OR) and $95 \%$ confidence intervals ( $95 \% \mathrm{CI}$ ) for preeclampsia according to quartiles of selected fatty acids (FA). Reference group is defined as those in quartile 1. OR and (95\% CI) are adjusted for maternal age, parity, mid-arm circumference and gestational age at delivery.

erythrocyte levels among the top two quartiles of the distribution of 10-trans octadecanoic acid $\left(\mathrm{C}_{18: \ln 8 t}\right)$, compared with values in the lowest quartile was associated with an almost doubling in preeclampsia risk. The adjusted odds ratios for the third and fourth quartiles were 2.19 and 1.93, respectively (adjusted p-for trend $=$ 0.02). We observed a strong positive linear relationship between diunsaturated fatty acids with a trans double bond and risk of preeclampsia. After adjusting for confounding factors, women with successively increasing levels of 9-cis 12-trans octadecanoic acid $\left(\mathrm{C}_{18: 2 \mathrm{n} 6 \mathrm{ct}}\right)$ experienced a 2.50-fold, 3.11-fold and 3.02-fold increased risk of preeclampsia as compared to women in the lowest quartile $(\mathrm{OR}=2.5,95 \%$ CI 1.15-5.40; $\mathrm{OR}=3.11,95 \%$ CI 1.45 ; OR $=3.02,95 \%$ CI 1.41-6.45, respectively, adjusted $\mathrm{p}$ for trend $=0.01$ ). A similarly strong linear trend of increasing risk of preeclampsia was seen for increasing maternal erythrocyte 9-trans 12-cis octadecanoic acid $\left(\mathrm{C}_{18: 2 n \text { otc }}\right)$ levels.

\section{Discussion}

In this case-control study of pregnant women from Harare, Zimbabwe, we found little support for the hypothesized inverse association between omega-3 fatty acids and preeclampsia risk. A modest inverse association was seen for omega- 6 fatty acids and risk of preeclampsia. There was some evidence suggestive of a weak positive association between increasing levels of arachidonic acid and risk of preeclampsia. Overall, maternal erythrocyte $\mathrm{C}_{18}$ monounsaturated trans fatty acids were positively associated with preeclampsia risk. We observed a strong linear trend of increasing risk of preeclampsia associated with increasing levels of diunsaturated fatty acids with a trans double bond.

Several caveats must be considered when interpreting the results of our study. Statistical control for confounding factors resulted in modest changes in estimated odds ratios. Control for confounding may be incomplete because of random errors in measurements of covariates or errors in multivariate model specification. Additionally, we cannot exclude the possibility that our results are in part confounded by unmeasured factors such as maternal insulin resistance, gestational diabetes mellitus, and intake of salt. Glucose tolerance testing was not routinely performed and is considered to be uncommon in our study setting. We did not have information concerning maternal salt intake, thus we cannot exclude confounding as a possible limitation of our research. 
Table 3. Odds Ratios (OR) and $95 \%$ Confidence Intervals (CI) of preeclampsia according to quartile of percentages of omega- 6 polyunsaturated fatty acids in maternal erythrocytes, Harare, Zimbabwe, 1995-1996.

\begin{tabular}{lllccc} 
& \multicolumn{5}{c}{ Quartile of Measurement } \\
\cline { 2 - 5 } Fatty Acid & 1 (Low) & 2 & 3 & 4 (High) & $\begin{array}{l}\text { trend } \\
\text { p-value }\end{array}$
\end{tabular}

\section{Linoleic acid $\left(C_{18: 2 n \sigma}\right)$}

\begin{tabular}{|c|c|c|c|c|c|}
\hline Mean & 9.09 & 10.36 & 11.12 & 12.36 & \\
\hline No. cases & 69 & 35 & 29 & 37 & \\
\hline No. controls & 47 & 46 & 46 & 46 & \\
\hline OR & 1 & 0.52 & 0.43 & 0.55 & 0.02 \\
\hline $\operatorname{Adj} O R^{*}(95 \% C I)$ & 1 & $0.83(0.43-1.61)$ & $0.65(0.32-1.30)$ & $0.70(0.36-1.37)$ & 0.23 \\
\hline \multicolumn{6}{|c|}{$\gamma$ Linoleic acid $\left(C_{18: 3 n \sigma}\right)$} \\
\hline Mean & 0.03 & 0.26 & 0.32 & 0.38 & \\
\hline No. cases & 42 & 42 & 49 & 37 & \\
\hline No. controls & 47 & 47 & 45 & 46 & \\
\hline$O R$ & 1 & 1.00 & 1.22 & 0.90 & 0.93 \\
\hline Adj $O R^{*}(95 \% C I)$ & 1 & $0.87(0.44-1.71)$ & $1.23(0.64-2.39)$ & $0.96(0.48-1.90)$ & 0.83 \\
\hline \multicolumn{6}{|c|}{ Eicosadienoic acid $\left(C_{20: 2 n \sigma}\right)$} \\
\hline Mean & 0.39 & 0.46 & 0.51 & 0.59 & \\
\hline No. cases & 60 & 48 & 35 & 27 & \\
\hline No. controls & 47 & 46 & 46 & 46 & \\
\hline$O R$ & 1 & 0.82 & 0.60 & 0.46 & $<0.01$ \\
\hline Adj $O R^{*}(95 \% C I)$ & 1 & $1.12(0.59-2.12)$ & $0.75(0.38-1.46)$ & $0.58(0.29-1.17)$ & 0.08 \\
\hline \multicolumn{6}{|c|}{ Eicosatrienoic acid $\left(C_{20: 3 n}\right)$} \\
\hline Mean & 1.2 & 1.36 & 1.48 & 1.7 & \\
\hline No. cases & 52 & 41 & 39 & 38 & \\
\hline No. controls & 47 & 46 & 46 & 46 & \\
\hline OR & 1 & 0.81 & 0.77 & 0.75 & 0.31 \\
\hline Adj $O R^{*}(95 \% C I)$ & 1 & $0.69(0.36-1.34)$ & $0.77(0.39-1.50)$ & $0.53(0.27-1.03)$ & 0.09 \\
\hline \multicolumn{6}{|c|}{ Arachidonic acid $\left(C_{20: 4 n}\right)$} \\
\hline Mean & 11.58 & 12.43 & 13.06 & 13.86 & \\
\hline No. cases & 41 & 45 & 32 & 52 & \\
\hline No. controls & 47 & 46 & 46 & 46 & \\
\hline$O R$ & 1 & 1.12 & 0.80 & 1.30 & 0.58 \\
\hline Adj $O R^{*}(95 \% C I)$ & 1 & $1.19(0.60-2.33)$ & $0.79(0.29-1.59)$ & $1.29(0.66-2.54)$ & 0.71 \\
\hline
\end{tabular}

An additional important limitation of our study relates to the fact that maternal erythrocyte fatty acid composition was assessed following the diagnosis of preeclampsia. As a result, it is not possible to determine whether the differences are a cause or consequence of the disorder. Prospective studies, which allow the simultaneous measurement of dietary intake of fatty acids, as well as biochemical assessment of fatty acids status in a cohort of pregnant women followed longitudinally, and which include serial measurements of maternal systolic, diastolic and mean arterial pressures throughout pregnancy, will resolve these issues.

To the best of our knowledge, this is the first study to evaluate maternal fatty acid profiles of women from Zimbabwe. In general, mean erythrocyte levels of omega-3 fatty acids for normotensive control subjects in this population were lower than values seen for normotensive pregnant women in Seattle (Washington, 
Table 3. (Cont.) Odds Ratios (OR) and $95 \%$ Confidence Intervals (CI) of preeclampsia according to quartile of percentages of omega-6 polyunsaturated fatty acids in maternal erythrocytes, Harare, Zimbabwe, 1995-1996.

\begin{tabular}{|c|c|c|c|c|c|}
\hline \multirow[b]{2}{*}{ Fatty Acid } & \multicolumn{4}{|c|}{ Quartile of Measurement } & \multirow[b]{2}{*}{ trend p-value } \\
\hline & 1 (Low) & 2 & 3 & 4 (High) & \\
\hline \multicolumn{6}{|c|}{ Docosatetraenoic acid $\left(C_{22: 4 n 6}\right)$} \\
\hline Mean & 3.3 & 3.80 & 4.17 & 4.66 & \\
\hline No. cases & 51 & 53 & 36 & 30 & \\
\hline No. controls & 47 & 46 & 46 & 46 & \\
\hline$O R$ & 1 & 1.06 & 0.72 & 0.60 & 0.05 \\
\hline $\operatorname{Adj} O R^{*}(95 \%$ CI $)$ & 1 & $1.17(0.62-2.21)$ & $0.55(0.28-1.09)$ & $0.58(0.29-1.17)$ & 0.04 \\
\hline \multicolumn{6}{|c|}{ Sum Omega-6 fatty acids } \\
\hline Mean & 27.93 & 29.31 & 30.09 & 31.35 & \\
\hline No. cases & 64 & 44 & 34 & 28 & \\
\hline No. controls & 47 & 45 & 47 & 46 & \\
\hline OR & 1 & 0.72 & 0.53 & 0.45 & $<0.01$ \\
\hline $\operatorname{Adj} O R^{*}(95 \%$ CI $)$ & 1 & $0.85(0.45-1.61)$ & $0.66(0.35-1.33)$ & $0.46(0.23-0.92)$ & 0.02 \\
\hline \multicolumn{6}{|c|}{ Ratio of Omega-3/Omega 6} \\
\hline Mean & 0.20 & 0.22 & 0.24 & 0.29 & \\
\hline No. cases & 31 & 23 & 56 & 60 & \\
\hline No. controls & 47 & 45 & 47 & 46 & \\
\hline OR & 1 & 0.77 & 1.81 & 1.98 & $<0.01$ \\
\hline $\operatorname{Adj} O R^{*}(95 \%$ CI $)$ & 1 & $0.96(0.45-2.05)$ & $1.77(0.91-3.44)$ & $1.47(0.75-2.89)$ & 0.12 \\
\hline \multicolumn{6}{|c|}{ Ratio of Omega-3/Arachidonic acid } \\
\hline Mean & 0.46 & 0.53 & 0.58 & 0.66 & \\
\hline No. cases & 36 & 49 & 23 & 62 & \\
\hline No. controls & 46 & 46 & 46 & 47 & \\
\hline$O R$ & 1 & 1.36 & 0.64 & 1.69 & 0.24 \\
\hline Adj $O R^{*}(95 \% C I)$ & 1 & $1.01(0.37-1.73)$ & $0.39(0.18-0.83)$ & $1.13(0.58-2.19)$ & 0.87 \\
\hline
\end{tabular}

${ }^{*}$ Adjusted for maternal age, parity, mid-arm circumference and gestational age at delivery.

USA). The average percentage of eicosapentaenoic, docosapentaenoic and docosahexaenoic fatty acids in maternal erythrocytes were $70 \%, 15 \%$ and $12 \%$ lower, respectively, for women in Harare as compared with women in Seattle (Williams et al. 1995). The comparatively low average percentage of omega-3 fatty acids in erythrocytes of Zimbabwean women may have served to limit variability between individuals, and therefore reduce available statistical power to detect an association with preeclampsia risk (if indeed one exists in this study population). For omega- 6 fatty acids, women from Zimbabwe had $24 \%$ higher linoleic acid, $4 \%$ lower arachidonic acid, and $23 \%$ higher docosatetraeonic acid as compared to women from the USA.

Comparisons of our results with those from previous investigations are limited to contrasting unadjusted case-control group mean differences for omega- 3 and omega- 6 fatty acids. Some of our observed mean differences in maternal fatty acids between cases and controls in the present study are in general agreement with results from previous investigations. An earlier study of predominately white women in Seattle (Washington, USA) found that low erythrocyte levels of omega-3 fatty acids and high levels of omega- 6 fatty acids, particularly arachidonic acid, were associated with an increased risk of preeclampsia (Williams et al. 1995). Ogburn et al. (1984), also found some evidence of high levels of the omega- 6 fatty acid, arachidonic acid in preeclamptic as compared to normotensive Caucasian pregnant women. In their study of 11 preeclamptic cases and 19 controls, the investigators reported that maternal arachidonic acid levels, measured in plasma phospholipids, were $13.5 \%$ higher for cases compared with controls (Ogburn et al. 1984). However, our observations are at variance with reports from other investigators (Wang et al. 1991, Al et. al. 1995). Unlike Wang et al. (1991), who reported that plasma levels of arachidonic acid were reduced in preeclamptic women as compared with controls (11.4 \pm 1.0 and 12.8 \pm 0.9 , respectively) in the United States, we found the average 
Table 4. Odds Ratios (OR) and $95 \%$ Confidence Intervals (CI) of preeclampsia according to quartile of percentages of trans fatty acids in maternal erythrocytes, Harare, Zimbabwe, 1995-1996.

\begin{tabular}{|c|c|c|c|c|c|}
\hline \multirow[b]{2}{*}{ Fatty Acid } & \multicolumn{4}{|c|}{ Quartile of Measurement } & \multirow[b]{2}{*}{$\begin{array}{l}\text { trend } \\
\text { p-value }\end{array}$} \\
\hline & 1 (Low) & 2 & 3 & 4 (High) & \\
\hline \multicolumn{6}{|c|}{ 8,7,6-trans octadeceonic acid $\left(C_{18: \ln 10-12 t}\right)$} \\
\hline Mean & 0.04 & 0.05 & 0.07 & 0.11 & \\
\hline No. cases & 27 & 41 & 47 & 55 & \\
\hline No. controls & 47 & 46 & 46 & 46 & \\
\hline OR & 1 & 1.55 & 1.78 & 2.08 & 0.02 \\
\hline $\operatorname{Adj} O R^{*}(95 \% C I)$ & 1 & $1.33(0.64-2.75)$ & $1.37(0.68-2.80)$ & $1.63(0.81-3.26)$ & 0.19 \\
\hline \multicolumn{6}{|c|}{ 9-trans octadecanoic acid $\left(C_{18: \ln 9 t}\right)$} \\
\hline Mean & 0.08 & 0.12 & 0.16 & 0.23 & \\
\hline No. cases & 32 & 34 & 46 & 58 & \\
\hline No. controls & 47 & 46 & 48 & 44 & \\
\hline$O R$ & 1 & 1.09 & 1.41 & 1.94 & 0.02 \\
\hline $\operatorname{Adj} O R^{*}(95 \% C I)$ & 1 & $1.00(0.49-2.05)$ & $1.05(0.52-2.12)$ & $1.64(0.83-3.24)$ & 0.14 \\
\hline \multicolumn{6}{|c|}{ 10-trans octadecanoic acid $\left(C_{18: \ln 8 t}\right)$} \\
\hline Mean & 0.07 & 0.10 & 0.13 & 0.20 & \\
\hline No. cases & 25 & 25 & 61 & 59 & \\
\hline No. controls & 47 & 46 & 46 & 46 & \\
\hline$O R$ & 1 & 1.02 & 2.49 & 2.41 & $<0.01$ \\
\hline $\operatorname{Adj}$ OR $(95 \%$ CI $)$ & & $1.04(0.48-2.26)$ & $2.19(1.08-4.42)$ & $1.93(0.96-3.91)$ & 0.02 \\
\hline \multicolumn{6}{|c|}{ 11-trans octadecanoic acid $\left(C_{18: \ln 7 t}\right)$} \\
\hline Mean & 0.13 & 0.19 & 0.23 & 0.29 & \\
\hline No. cases & 36 & 41 & 44 & 49 & \\
\hline No. controls & 47 & 47 & 45 & 46 & \\
\hline OR & 1 & 1.14 & 1.28 & 1.39 & 0.25 \\
\hline $\operatorname{Adj} O R^{*}(95 \% C I)$ & 1 & $0.83(0.42-1.65)$ & $1.08(0.57-213)$ & $1.07(0.54-2.12)$ & 0.66 \\
\hline \multicolumn{6}{|c|}{ 12-trans octadecanoic acid $\left(C_{18: \ln 6 t}\right)$} \\
\hline Mean & 0.2 & 0.24 & 0.27 & 0.34 & \\
\hline No. cases & 31 & 35 & 50 & 54 & \\
\hline No. controls & 48 & 45 & 46 & 46 & \\
\hline OR) & 1 & 1.2 & 1.68 & 1.82 & 0.03 \\
\hline $\operatorname{Adj} O R^{*}(95 \% C I)$ & 1 & $0.92(0.45-1.91)$ & $1.22(0.61-2.45)$ & $1.29(0.65-2.58)$ & 0.33 \\
\hline \multicolumn{6}{|c|}{ Sum Monounsaturated trans fatty acids } \\
\hline Mean & 0.54 & 0.71 & 0.86 & 1.13 & \\
\hline No. cases & 30 & 33 & 52 & 55 & \\
\hline No. controls & 47 & 46 & 46 & 46 & \\
\hline OR) & 1 & 1.12 & 1.77 & 1.87 & 0.02 \\
\hline $\operatorname{Adj} O R^{*}(95 \% C I)$ & 1 & $1.55(0.78-3.08)$ & $1.41(0.71-2.81)$ & $1.19(0.58-2.47)$ & 0.19 \\
\hline
\end{tabular}

percent of arachidonic acid in maternal erythrocyte to be slightly increased in Zimbabwean women with preeclampsia. Van der Schouw et al. (1991), also reported higher percent plasma phospholipid arachidonic acid in Dutch women with non-proteinuric pregnancy induced hypertension as compared with normotensive controls $(9.1 \pm 0.5$ vs. $8.7 \pm 0.4)$.

Few investigators have studied systematically maternal dietary intake of fatty acids during pregnancy. Results from studies conducted among pregnant women from the Faroes islands and mainland Danish women suggest that diets high in omega-3 fatty acids are associated with prolonged gestation, increased birth weight and a reduction in risk of preeclampsia (Olsen et al. 1991). Crawford et al. (1986) reported that diets low in omega-3 fatty acids (as reflected by maternal reports) 
Table 4. (Cont.) Odds Ratios (OR) and $95 \%$ Confidence Intervals (CI) of preeclampsia according to quartile of percentages of trans fatty acids in maternal erythrocytes, Harare, Zimbabwe, 1995-1996.

\begin{tabular}{lllccl}
\hline & \multicolumn{5}{c}{ Quartile of Measurement } \\
\cline { 2 - 5 } Fatty Acid & 1 (Low) & 2 & 3 & 4 (High) & $\begin{array}{l}\text { trend } \\
\text { p-value }\end{array}$ \\
\hline
\end{tabular}

\begin{tabular}{|c|c|c|c|c|c|}
\hline \multicolumn{6}{|c|}{ 9-cis 12-trans octadecanoic acid $\left(C_{18: 2 n 6 c t}\right)$} \\
\hline Mean & 0.03 & 0.04 & 0.05 & 0.08 & \\
\hline No. cases & 20 & 44 & 52 & 54 & \\
\hline No. controls & 47 & 46 & 46 & 46 & \\
\hline OR) & 1 & 2.25 & 2.66 & 2.76 & $<0.01$ \\
\hline $\operatorname{Adj} O R^{*}(95 \% C I)$ & 1 & $2.50(1.15-5.40)$ & $3.11(1.45-6.67)$ & $3.02(1.41-6.45)$ & 0.01 \\
\hline \multicolumn{6}{|c|}{ 9-trans 12-cis octadecanoic acid (C $\left.C_{18: 2 n b t c}\right)$} \\
\hline Mean & 0.02 & 0.03 & 0.04 & 0.05 & \\
\hline No. cases & 15 & 41 & 38 & 76 & \\
\hline No. controls & 47 & 46 & 46 & 46 & \\
\hline OR & 1 & 2.79 & 2.59 & 5.18 & $<0.01$ \\
\hline $\operatorname{Adj} O R^{*}(95 \% C I)$ & 1 & $1.53(0.68-3.44)$ & $2.11(0.95-4.69)$ & $3.32(1.55-7.13)$ & $<0.01$ \\
\hline \multicolumn{6}{|c|}{ Sum Diunsaturated trans fatty acids } \\
\hline Mean & 0.05 & 0.07 & 0.08 & 0.12 & \\
\hline No. cases & 17 & 31 & 52 & 70 & \\
\hline No. controls & 48 & 45 & 46 & 46 & \\
\hline$O R$ & 1 & 1.95 & 3.19 & 4.30 & $<0.01$ \\
\hline Adj $O R^{*}(95 \% C I)$ & 1 & $1.52(0.67-3.44)$ & $3.56(1.65-7.66)$ & $3.39(1.60-7.17)$ & $<0.01$ \\
\hline
\end{tabular}

${ }^{*}$ Adjusted for maternal age, parity, mid-arm circumference and gestational age at delivery.

were associated with low birth weight and smaller head circumference. However, Harper et al. (1991) reported that birth weights were similar for Orcadian women, who are reported to eat $30 \%$ more fish than the Aberdonians. The latter study did not measure individual dietary intake. Using a simple, un-validated food frequency questionnaire, Atkinson et al. (1998) reported that maternal consumption of kapenta (a small white fish) was associated with a modest, though statistically nonsignificant, reduction in risk of preeclampsia. Importantly, maternal reported intake of other fish (e.g. bream etc.) was not associated with the outcome. $\mathrm{Al}$ and colleagues (1995) using a food frequency questionnaire, measured maternal dietary intake for 29 women with pregnancy-induced hypertension and an equal number of controls from the Maastricht region of the Netherlands, observed no differences in maternal consumption of total, saturated, monounsaturated and polyunsaturated fats, proteins or total calories.

Differences in population characteristics such as racial distributions, as well as dissimilar distributions of the severity of preeclampsia studied could account for some of the variation in studied results. Some investigators included pregnancy-induced hypertension without proteinuria in their case group. Pregnancyinduced hypertension with proteinuria (preeclampsia) and without proteinuria (gestational hypertension) may differ considerably in etiologic and epidemiologic characteristics. Other possible explanations for the heterogeneity in results from the few studies involving the biochemical assessment of maternal fatty acid status in relation to risk of preeclampsia include: 1) small sample size yielding statistically unstable point estimates, 2) whether fatty acids were measured in plasma phospholipids or in the membranes of erythrocytes, 3) biochemical techniques used to measure fatty acids, and 4) statistical analytical techniques used to summarize fatty acids profiles. Importantly, previous investigators have generally not controlled for potential confounding factors, nor have they reported the magnitude of 
association between preeclampsia and varying levels of fatty acids.

High levels of elaidic acid (a trans fatty acid) in maternal erythrocytes have been found to be associated with a significant increase in risk of preeclampsia (Williams et al. 1998). Women with the highest level of elaidic acid (median $=0.47$ ) were 7.4 times more likely to have had their pregnancy complicated by preeclampsia $(\mathrm{OR}=7.4,95 \%$ CI 1.4-39.7) as compared with those women with the lowest levels (median $=0.24$ ). Results of the present study which allowed for the separation of $\mathrm{C}_{18}$ monounsaturated trans fatty acids and diunsaturated fatty acids with a trans double bond, respectively are in general agreement with this earlier observation. We know of no other study which evaluates risk of preeclampsia in relation to biochemical markers of maternal trans fatty acids profiles.

The finding that trans fatty acids, more than other fatty acids, promote modifications of plasma lipids in favor of an atherogenic profile (Katan and Zock 1995) suggest a possible physiological mechanism for the observed association of trans fatty acids with risk of preeclampsia. Dietary trans fatty acids are associated with a reduction in plasma high density cholesterol concentrations, and elevations in triglyceride and lipoprotein (a) concentrations. Hyperlipidemia, particularly hypertriglyceridemia, has been consistently reported to be associated with preeclampsia (Enquobahrie et al. 2004). In addition, lipoprotein (a) has been shown to accumulate in the placental arterial of women with preeclampsia (Meekins et al. 1994). Hence, there remains the possibility that the influence of trans fatty acids on risk of preeclampsia may, in part, be mediated by their effects on maternal plasma lipid and lipoprotein profile. Trans fatty acids may also exert their influence on preeclampsia through their ability to inhibit the elongation and saturation of essential fatty acids (Kinsella et al. 1981, Carlson et al. 1997). Limited available data suggest that trans fatty acids may compete with essential fatty acids, particularly in the context of diets deficient in essential fatty acids (Carlson et al. 1997). Importantly, the requirement for essential fatty acids is increased during pregnancy (Holman et al. 1991). Hence, taken together several studies suggest possible plausible biological mechanisms that may underlie the statistical associations observed in our study. However, additional epidemiological and clinical studies which characterize fatty acids and plasma lipid profiles in preeclamptic and normotensive pregnant women are needed to more rigorously re-evaluate these hypotheses.

In summary, we found that omega-3 fatty acids are comparatively lower among pregnant Zimbabwean women as compared with pregnant North American women. Our results provide little support for the hypothesized inverse relation between omega-3 fatty acids and risk of preeclampsia. To the best of our knowledge, we are the first to provide empirical evidence suggestive of a positive association between maternal trans fatty acid status and risk of preeclampsia in a black African population. This observation corroborates and extends an earlier finding of a strong linear increase in risk for preeclampsia associated with elaidic acid a trans fatty acid. Residual confounding cannot be entirely excluded, nor we can with confidence attribute observed associations exclusively to differences in maternal dietary patterns (preeclampsia-associated alterations in fatty acid metabolism remain a possible explanation for these cross-sectional data). Additional prospective studies which simultaneously evaluate maternal intake of fats and other nutrients (using information from dietary intake questionnaires and biochemical markers) are needed to better characterize the role that maternal dietary characteristics play in the etiology and pathophysiology of preeclampsia.

\section{Acknowledgements}

This research was supported in part by awards from the National Institutes of Health, Fogarty International Center (R03-TW-00981 and T37-TW-00049), and the National Institute of Child Health and Human Development (R0132562).

\section{References}

AL MD, HOUWELINGEN ACV, BASART-SMOOK A, HASAART TH, ROUMEN FJ, HONSTRA G: The essential fatty acid status of mother and child in pregnancy-induced hypertension: a prospective longitudinal study. Am J Obstet Gynecol 172: 1605-1614, 1995.

AMERICAN COLLEGE OF OBSTETRICIANS AND GYNECOLOGISTS: Hypertension in pregnancy. ACOG Technical Bulletin 219: 1-8, 1996. 
ARO A, KARDINAAL AFM, SALMINEN I, KARK JD, RIEMERSMA RA, DELGADO-RODRIGUEZ M, GOMEZARACENA J, HUTTUNEN JK, KOHLMERIER L, MARTIN BC: Adipose tissue isomeric trans fatty acids and risk of myocardial infarction in nine countries: the EURAMIC study. Lancet 345: 273-278, 1995.

ATKINSON JO, MAHOMED K, WILLIAMS MA, WOELK GB, MUDZAMIRI S, WEISS NS: Dietary risk factors for preeclampsia among Zimbabwean women. Cent Afr J Med 44: 86-92, 1998.

CARLSON SE, CLANDININ MT, COOK HW, EMKEN EA, FILER LJ, JR: Trans fatty acids: infant and fetal development. Am J Clin Nutr 66: 717S-736S 1997.

CLAUSEN T, SLOTT M, SOLVOLL K, DREVON CA, VOLLSET SE, HENRIKSEN T: High intake of energy, sucrose, and polyunsaturated fatty acids is associated with increased risk of preeclampsia. Am J Obstet Gynecol 185: 451-458, 2001.

CRAWFORD MA, DOYLE W, CRAFT IL, LAURENCE BM: A comparison of food intake during pregnancy and birth weight in high and low socioeconomic groups. Prog Lipid Res 25: 249-254, 1986.

ENQUOBAHRIE DA, WILLIAMS MA, BUTLER C, FREDERICK IO, MILLER RS, LUTHY DA: Maternal plasma lipid concentrations in early pregnancy and risk of preeclampsia. Am J Hypertens 17: 574-581 2004.

FARQUHAR JW, AHRENS EH, JR: Effects of dietary fats on human erythrocyte fatty acid patterns. J Clin Invest $\mathbf{4 2}$ : 675-685 1983.

HARPER V, MACINNES R, CAMPBELL D, HALL: Increased birth weight in northerly islands: is fish consumption a red herring? BMJ 303: 166, 1991.

HARRIS WS: Extending cardiovascular benefits of omega-3 fatty acids. Curr Atherosclerosis Rep 57: 375-380, 2005.

HOLMAN RT, JOHNSON SB, OGBURN PL: Deficiency of essential fatty acids and membrane fluidity during pregnancy and lactation. Proc Natl Acad Sci 88: 4835-4839, 1991.

HSIEH CC, MAISONNEUVE P, BOYLE P, MACFARLANE GJ, ROBERTSON C: Analysis of quantitative data by quantiles in epidemiologic studies: classification according to cases, noncases or all subjects. Epidemiology $\mathbf{2}$ : 137-140, 1991.

HU FB, STAMPFER MJ, MANSON JE, RIMM E, COLDITZ GA, ROSNER BA, HENNEKENS CH, WILLETT WC: Dietary fat intake and the risk of coronary heart disease in women. N Engl J Med 337: 1491-1499, 1997.

KATAN MB, ZOCK PL: Trans fatty acids and their effects on lipoproteins in humans. Annu Rev Nutr 15: 473-493, 1995.

KESTIN A, CLIFTON P, BELLING GB, NESTEL PJ: N-3 fatty acids of marine origin lower systolic blood pressure and triglycerides but raise LDL cholesterol compared to n-3 and n-6 fatty acids from plants. Am J Clin Nutr 51: 1028-1034 1990.

KINSELLA JE, BRUCKNER G, MAI J, SHIMP J: Metabolism of trans fatty acids with emphasis on the effects of trans, trans-octadecadienoate on lipid composition, essential fatty acid, and prostaglandins: an overview. Am J Clin Nutr 34: 2307-2318, 1981.

KINSELLA JE, LOKESH B, STONE RA: Dietary omega-3 polyunsaturated fatty acids and amelioration of cardiovascular disease: possible mechanisms. Am J Clin Nutr 52: 1-28 1990.

KOLETZKO B: Trans fatty acids may impair biosynthesis of long-chain polyunsaturates and growth in man. Acta Paediatr 81: 302-306, 1992.

KRASOVECK K, ANDERSON MA FOR THE USAID/WHO/PAHO MOTHERCARE: Maternal anthropometry for prediction of pregnancy outcomes: Memorandum from USAID/WHO/PAHO MotherCare meeting. Bull WHO 69: 523-532, 1991.

LEPAGE G, ROY CC. Direct transesterification of all lipids in a one-step reaction. J Lipid Res 27: 114-120, 1986.

MEEKINS JW, PIJNENBORG R, HANSSENS M, VAN ASSCHE A, MCFADYEN IR: Immunohistochemical detection of lipoprotein(a) in the wall of placenta, bed spiral arteries in normal and severe pre-eclamptic pregnancies. Placenta 15: 511-524, 1994.

OGBURN PL, WILLIAMS PP, JOHNSON SB, HOLMAN RT: Serum arachidonic acid levels in normal and preeclamptic pregnancies. Am J Obstet Gynecol 148: 5-9, 1984. 
OLSEN SF, HANSEN HS, SOMMER S, JENSEN B, SORENSEN TIA, SECHER NJ, ZACHARIAASEN P: Gestational age in relation to marine omega- 3 fatty acids in maternal erythrocytes: a study of women in the Faroe Islands and Denmark. Am J Obstet Gynecol 164: 1203-1209, 1991.

QIU C, PHUNG TTT, VADACHKORIA S, MUY-RIVERA M, SANCHEZ SE, WILLIAMS MA: Oxidized lowdensity lipoprotein (Oxidized LDL) and the risk of preeclampsia. Physiol Res 55: 491-500, 2006.

ROBERTS JM, PEARSON G, CUTLER J, LINDHEIMER M: Summary of the NHLBI Working Group on research on hypertension during pregnancy. Hypertension 41: 437-445, 2003.

ROBERTS TL, WOOD DA, REIMERSMA RA, GALLAGHER PJ, LAMPE FC: Trans isomers of oleic and linoleic acids in adipose tissue and sudden cardiac death. Lancet 345: 278-282, 1995.

ROTHMAN KJ, GREENLAND S: Modern Epidemiology. Lippincott-Raven Publishers, Philadelphia, 1998, pp 311316.

VAN DER SCHOUW YT, AL MDM, HORNSTRA G, BULSTRA-RAMAKERS MTEW, HUISJES HJ: Fatty acid composition of serum lipids of mothers and their babies after normal and hypertensive pregnancies. Prostaglandins Leukot Essent Fatty Acids 44: 247-252, 1991.

WANG Y, KAY HH, KILLAM AP: Decreased levels of polyunsaturated fatty acids in preeclampsia. Am J Obstet Gynecol 164: 812-818, 1991.

WILLIAMS MA, MITTENDORF R: Maternal Morbidity. In: Women and Health. GOLDMAN MB, HATCH M (eds). San Diego, Academic Press, 2000, pp.172-181.

WILLIAMS MA, ZINGHEIM RW, KING IB, ZEBELMAN AM: Omega-3 fatty acids in maternal erythrocytes and risk of preeclampsia. Epidemiology 6: 232-237, 1995.

WILLIAMS MA, KING IB, SORENSEN TK, ZINGHEIM RW, TROYER BL, ZEBELMAN AM, LUTHY DA: Risk of preeclampsia in relation to elaidic acid (trans fatty acid) in maternal erythrocytes. Gynecol Obstet Invest 46: 84-87, 1998.

\section{Reprint requests}

Michelle A. Williams, Department of Epidemiology, MIRT Program, 1959 NE Pacific Street, HSB-F263 (Box 357236), Seattle, WA 98195, USA. E-mail: Mwilliam@u.washington.edu 